\title{
Vincent McKoy: pioneer of computational electron-molecule scattering and photoionization
}

\author{
Carl Winstead ${ }^{\mathrm{a}}$ (D) \\ 4475 Ravine Drive, Westerville OH 43081, USA
}

Received 16 December 2021 / Accepted 22 December 2021 / Published online 14 January 2022 (C) The Author(s), under exclusive licence to EDP Sciences, SIF and Springer-Verlag GmbH Germany, part of Springer Nature 2022

\begin{abstract}
Vincent McKoy - Vince to his friends and colleagueswas a pioneer in the theoretical study of free electrons interacting with molecules. Over several decades, he and his collaborators worked to build and apply the computational machinery for accurate and informative calculations of molecular photoionization processes and of electron collisions with neutral molecules, with a sustained focus on extending the capabilities of that machinery to ever more complex targets while maintaining close contact with experimentalists and with those interested in applications. What began in the 1970s with studies of diatomic targets of atmospheric interest thus arrived, by the 2010s, at the study of complex polyatomic molecules important in biology, work that is carried on by his former students and postdocs today.
\end{abstract}

Vince was born in the British West Indies in 1938 and graduated from the Technical University of Nova Scotia, now part of Dalhousie University, in 1960, with a B. Sc. in chemical engineering. Four years later, he received his $\mathrm{Ph}$. D. in chemistry from Yale, where his dissertation, under Oktay Sinanoğlu, addressed dissociation pressures of gas hydrates. In that same year (1964), he arrived at Caltech, where he would remain until declining health compelled his retirement as Professor of Theoretical Chemistry in 2016.

Caltech in the 1960 s and 1970 s was a stimulating environment for chemical physics. The Noyes Laboratory of Chemical Physics opened in 1967 and housed Vince's theoretical group alongside the group of William Goddard III, focused on bound-state electronic structure, and the group of Aron Kuppermann, which combined experimental and theoretical study of collision processes. In this environment, Vince's interests evolved from electronically excited bound states of molecules to continuum states. Instrumental to this evolution were Thomas Rescigno, who was a postdoc in the McKoy group from 1973 to 1975, and William McCurdy, who received his Ph. D. with Vince in 1976. As readers will know, Tom and Bill themselves went

\footnotetext{
a e-mail: carlwinstead@icloud.com (corresponding author)
}

on to become leading figures in theoretical electronmolecule scattering and molecular photoionization and continue to collaborate regularly.

As the 1970s gave way to the 1980s, Vince's group began to build practical computational machinery for low-energy electron-molecule scattering and photoionization. At this time (and for some time after), most scattering calculations relied on local model potentials to account for exchange and polarization, reducing the many-electron collision problem to a more tractable single-particle problem. Vince's intuition was that the future of the field lay with full-on treatments that accounted properly for exchange and polarization and that would also permit the study of electron impact excitation. Two talented graduate students, Robert Lucchese and Marco Lima, together with a remarkable postdoc, Kazuo Takatsuka, would help realize Vince's ambition. Bob developed a powerful computer code for photoionization studies based on the iterative solution of the Schwinger equation. Kazuo and Vince explored a variety of variational approaches to the electronmolecule collision problem and began developing code to implement what they called the Schwinger multichannel (SMC) method, work in which Marco joined and which led to the group's first publications with elastic and electronically inelastic cross sections for diatomic molecules. Bob went on to build a strong research program at Texas A\&M University, while Marco returned to Brazil and established a premier electron-molecule scattering program at the State University of Campinas (Unicamp), and Kazuo assumed a professorship at the University of Tokyo and established a vigorous and eclectic research program.

As the 1980s progressed, the focus of Vince's photoionization studies became resonance-enhanced multiphoton ionization, or REMPI. REMPI had emerged as a powerful spectroscopic method, but its utility was enhanced by collaboration with theoretical work that could help interpret the photoelectron angular distributions. Students including Richard Dubs, Matthew Braunstein, and Henrik Rudolph participated in this work. On the electron-molecule scattering side, gradu- 
ate student Howard Pritchard and postdocs Luiz Brescansin, Kazunori Watari, and Seido Nagano continued to study elastic and inelastic processes, including (in collaboration with Prof. Horia Metiu of the University of California, Santa Barbara) studies of scattering from molecules adsorbed on metal surfaces.

By the late 1980s, it was clear that accurate SMC calculations of electron scattering cross sections were possible, but it was equally clear that their computational cost was an obstacle. When the author joined the group as a postdoc in 1987, a major focus was accelerating the main computational step, the evaluation of the Green's function term in the SMC variational expression. This was accomplished, but even so, the rapid growth in computational expense with electron count threatened to preclude routine calculations on polyatomic targets. Faced with this barrier, Vince was intrigued by work going on at Caltech and at the Jet Propulsion Laboratory (JPL) on massively parallel computing. Geoffrey Fox of Physics had established the Caltech Concurrent Computation Project $\left(\mathrm{C}^{3} \mathrm{P}\right)$ to develop experimental hardware and some of the first system software for dividing up a problem among multiple processes running on different processors; JPL was building a series of prototype parallel computers based on a hypercube architecture; and scientists both on campus and at JPL, including Vince's Chemical Physics colleague Aron Kuppermann, were applying parallel computing to real scientific problems. Vince arranged for Paul Hipes, who had learned parallel programming in the course of his just-completed Ph. D. work in the Kuppermann group and was staying on for a short postdoc with $\mathrm{C}^{3} \mathrm{P}$, to work with the author, who by now knew the SMC code, to develop a parallel version. By the time Paul left, we had a mostly working code for elastic scattering in the static-exchange approximation that ran efficiently on JPL's Gamma hypercubes; further work resulted in a full-fledged code that handled polarization effects and inelastic processes and was ported to a succession of experimental and commercial parallel computers.

In the 1990s and early 2000s, Vince's research program focused on exploiting the potential of the computational tools that had been developed while continuing to evolve their capabilities. Kwanghsi Wang, who had joined the photoionization side of the group as a postdoc in the late 1980s and would remain as a staff scientist through Vince's retirement, generalized the photoionization code to treat nonlinear polyatomic molecules. The unique capabilities of the SMC scattering code were applied to a wide range of gases relevant to plasma etching of semiconductors; this work was done in collaboration with W. Lowell "Skip" Morgan, who brought expertise in the construction of selfconsistent cross section datasets. Thereafter, the focus of the electron scattering work shifted to the interaction of low-energy electrons with biomolecules, in particular the constituents of DNA, in response to the discovery by Léon Sanche and collaborators at Sherbrooke that dissociative electron attachment induces single- and double-strand breaks in DNA strands. Vince's calculations provided resonance assignments, including indications that some apparent shape resonances also contained core-excited character.

At the same time, a second scientific generation enriched the group. In succession, Márcio Bettega, Márcio Varella, and Sergio Sanchez earned their doctorates with Marco Lima at Unicamp and joined the McKoy group as postdocs studying electron-molecule collisions before founding their research programs in Brazil. On the photoionization side, Vince renewed collaboration with Kazuo Takatsuka, who had grown interested in nonadiabatic dynamics in molecular excited states. Time-delayed pump-probe spectroscopy, also known as time-resolved photoelectron spectroscopy (TRPES), was the natural experimental probe of such dynamics and was also a natural extension of the REMPI spectroscopy already being studied by the group. Yasuki Arasaki from Prof. Takatsuka's group was a repeat visitor to Caltech, working with Kwanghsi to develop computational treatments that encompassed both the electronic and nuclear dynamics involved in TRPES, with a particular focus on nonadiabatic interactions that coupled excited-state potential surfaces. Márcio Varella was also intimately involved in this work, holding postdoctoral appointments both with the Takatsuka group and again at Caltech before accepting a faculty position in Brazil.

Vince believed in the synergism between theory and experiment, and throughout his career he valued close collaboration with experimentalists. Many papers on electron-molecule scattering were joint publications with leading experimental groups, including those of Michael Allan in Switzerland, Michael Brunger and Stephen Buckman in Australia, Murtadha "Morty" Khakoo just down the road from Caltech in Fullerton, and Hiroshi Tanaka in Japan. In such collaborations, the goal was not just to test whether the calculation "worked" but to use it to enhance understanding of the measurement, for example by providing definitive assignments for observed resonances. Likewise, much of the TRPES work involved collaboration with the group of Albert Stolow in Canada and aimed to assist in the interpretation of complicated measurements by providing independent information on not only the energetics but also the photoelectron angular distributions associated with the different excited-state surfaces.

As much as he prized collaboration for its scientific value, Vince prized it equally for the occasion it afforded to interact informally with researchers around the world. Vince was a "people person," and he enjoyed travel and sampling other cultures. His especially strong long-term connection to Brazil is reflected not only in the many Brazilian students and postdocs that his group hosted but also in the frequent visits he made to Brazil, where he maintained experimental and theoretical collaborations until his retirement, and where he leaves a strong scientific legacy. 